
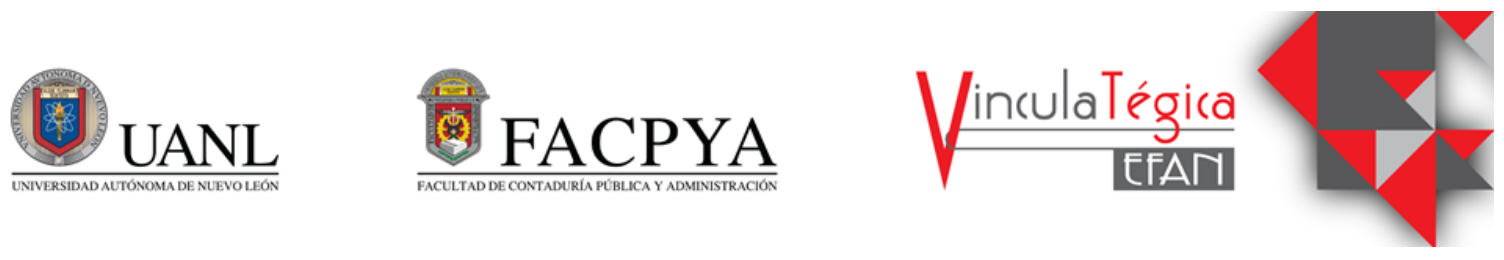

\title{
Factores que influyen en el emprendimiento de los alumnos de la rama administrativa y de negocios en la UANL
}

\author{
José Luis González Arizpe ${ }^{1}$, Paula Villalpando Cadena ${ }^{2}$ y José Barragán Codina ${ }^{3}$ \\ ${ }^{1}$ Profesor-investigador de la Facultad de Contaduría Pública y Administración, \\ jgonzaleza@uanl.edu.mx, San Nicolás de los Garza, Nuevo León, C.P. 66455. \\ ${ }^{2}$ Profesor-investigador de la Facultad de Contaduría Pública y Administración, \\ paula.villalpandocd@uanl.edu.mx, San Nicolás de los Garza, Nuevo León, C.P. 66455. \\ ${ }^{3}$ Profesor-investigador de la Facultad de Contaduría Pública y Administración, \\ jose.barragancdn@uanl.edu.mx, San Nicolás de los Garza, Nuevo León, C.P. 66455.
}

Información del artículo revisado por pares

Fecha de aceptación: junio-2021

Fecha de publicación en línea: diciembre-2021

DOI: https://doi.org/10.29105/vtga7.2-32

\begin{abstract}
Resumen
El emprendimiento en estos tiempos se ha presentado como una alternativa de ingreso única para gran parte de la población mexicana, desde personas con una carrera terminada, hasta aquellos que están en vías de finalizar alguna, esto va desde iniciar con pequeños negocios hasta los muy grandes con inversiones elevadas y con el fin de obtener un retorno y una estabilidad económica que en ocasiones no encuentran en trabajos jornadas promedio de 8 horas. Aunado a esto, se suman las diferentes situaciones que se están viviendo actualmente en el país, como el encarecimiento de servicios básicos (vivienda, canasta básica, etc.), desarrollo de nuevos productos que lucen inalcanzables para una gran mayoría de la población debido al coste del mismo y sumado al nivel socioeconómico en el que se podrían encontrar las familias y las personas que deseen adquirirlo y el más importante, a razón personal, no depender de alguien más para obtener recursos económicos que les permitan subsistir en una vida que, sin duda, se torna complicada conforme pasa el
\end{abstract}

\begin{abstract}
Entrepreneurship in current times has been presented as a single income alternative for a large part of a country population, this goes from persons with a bachelor's degree or the ones that are in their way to finish it, the activities go from starting small businesses to very large ones with high investments and to obtain a return and economic stability that sometimes does not They find in their day-to-day jobs. In addition to this, the different situations that are currently being experienced in the country are added, such as the increase in basic services (housing, basic basket, etc.), development of new products that look unattainable for a large majority of the population due to the cost of the same and added to the socioeconomic level in which families and people who wish to acquire it could be found and the most important, on a personal basis, not to depend on someone else to obtain economic resources that allow them to live in a life that, without doubt, it becomes complicated as time goes by. The present research work is focused on understanding not only what leads a person to undertake, but also the factors that are considered key or impact to generate that
\end{abstract}


tiempo. El presente trabajo de investigación está enfocado en entender no solo lo que conlleva a una persona a emprender, sino también los factores que se consideran clave o de impacto para generar ese impulso necesario para poder emprender y, sobre todo, llevarlo por un buen camino y que al final esta se geste de manera adecuada y la lleve al éxito esperado.

Palabras clave: emprendimiento, economía, crecimiento, necesidad, oportunidades.

\section{INTRODUCCIÓN}

En un mundo lleno de incertidumbre en materia social y sobre todo económica, las personas buscan de manera desesperada recursos que los ayuden a subsistir, desafortunadamente, la situación actual ha desacelerado en gran medida las fuentes de empleo, esto ha hecho a mucha gente voltear e incursionar en el emprendimiento, que, si bien ha sido explorado y abordado por muchos, no todos logran el éxito deseado.

Las nuevas generaciones no han estado exentas de esta nueva ola emprendedora, es por eso por lo que las universidades han apostado fuerte por incrementar en su malla curricular materias que permiten a los jóvenes adquirir el conocimiento necesario para aperturas o lanzamientos de nuevos negocios o productos a nivel nacional o internacional.

En la Universidad Autónoma de Nuevo León (UANL) esto no ha sido la excepción y a través de su escuela de negocios la Facultad de Contaduría Pública y Administración (FACPyA) ha impulsado por medio de su malla curricular durante años la formación de empresas, esto ha inculcado en muchos estudiantes el querer comenzar con un negocio propio, hay quien lo ha intentado y lo ha logrado con éxito, de esto se deriva la presente investigación, no solo el entender por qué los estudiantes de las escuelas de negocios emprenden $\mathrm{o}$ buscan emprender, si no comprender en su totalidad los detonantes que llevan a estos a decantarse por esta actividad, que creen que influye para que abandonen en ocasiones una idea tan arraigada en nuestra sociedad como lo es la estabilidad laboral y impulse to make an activity of a new business, and more important to take the practice of entrepreneurship on track and in a good path and that in the end this is managed in a way adequate and lead to the expected success.

Keywords: entrepreneurship, economy, growth, necessity, opportunity.

JEL: L26, L29.

aventurarse a algo nuevo, complejo y sobre todo desconocido para ellos.

\section{Antecedente teórico}

La actividad emprendedora de acuerdo con Melendrez (2018) es un concepto directamente relacionado con el desarrollo de nuevas estructuras empresariales que vienen a dar un impacto en las economías locales y nacionales. En el mismo sentido, Hernández y Arano (2015) sostienen que generan un efecto positivo en el desarrollo de nuevas fuentes de empleo e incluso en el incremento de riquezas propias y del país.

Ahora bien, el emprendimiento en México ha sido un detonante en los últimos años, pero a pesar de esto, aún falta mucho para poder considerar que esta práctica sea una de las mejores posicionadas en el país, esto se confirma mediante un dato importante del reporte de Monitoreo Global de Emprendimiento (GEM), elaborado por el Babson College y la London Business School (2018), este estudio muestra a México en el lugar 23 de 54 países analizados, solo estando dos peldaños debajo de Argentina que se encuentra en el lugar 21.

Evaluando los distintos puntos de opinión, podemos determinar que el emprendimiento es una actividad crucial no solo para quien lo ejecuta, sino también para el desarrollo económico de un país, por lo que el entender los factores que detonan esta práctica dentro de los jóvenes es necesario para gestionar y ofrecer la ayuda requerida para el que de soporte al aprovechamiento de quienes apenas comienzan con este tipo de práctica (Melendrez, 2018). 


\section{MARCO TEÓRICO}

De acuerdo con Álvarez, Giraldo y Martínez (2014) y Alvarado, Antonio y Ortiz (2020), el emprendimiento en la actualidad es un asunto de gran interés a nivel nacional e internacional, sobre todo por sus efectos sobre la economía y el desarrollo de negocios y como no serlo, tan solo imaginar que todas aquellas personas y principalmente los jóvenes pudieran llevar esta práctica con éxito vendrían a generar aportes importantes en los sectores económicos del país.

También, Minniti (2012) menciona que la figura del emprendedor ha sido reconocida por las grandes organizaciones a nivel internacional como el Banco Mundial (BM) o el Fondo Monetario Internacional (FMI) por las aportaciones que realizan a las economías de los países, es por eso que el análisis y comprensión de este tema, es de suma importancia y sobre todo trascendental a nivel global.

\section{Emprendimiento}

El emprendimiento es un tema que en los últimos años ha tomado una mayor relevancia debido al comportamiento y empeño de las nuevas generaciones de encontrar el trabajo perfecto que les dé un equilibrio en tiempo que muchos argumentan no consiguen el estando, trabajando para alguien más (Canedo, et al, 2014).

Marulanda y Morales (2016) definen al emprendimiento como la práctica que genera desarrollo económico y que funge como el motor de un país, esto se sustenta con datos estadísticos, ya que, de acuerdo al Instituto Nacional de Estadística Geográfica e Informática (INEGI), de 4 millones de unidades empresariales en México, 99.8\% son Pequeñas y Medianas Empresas (PyMES), por lo que es indudable pensar que no son un detonante para la economía de un país, sobre todo por el aporte que generan en el tema de generación de empleos y su aporte en relación a impuestos.

También hay quienes ven al emprendimiento como la solución a las problemáticas sociales que pudiera enfrentar un país y sirve como aliciente de superación para muchas personas, Staniewski (2016) define al emprendimiento como el antídoto para problemas económicos y sociales contemporáneos de una sociedad y que esta práctica sirve como alternativa a un empleo actual y principalmente como desarrollo profesional.

Al final y tomando de referencia la definición de Mezzina y Hochsztain (2015) se puede decir que la definición para este estudio de emprendimiento es que es una práctica que busca contribuir al desarrollo económico del país a través de la generación de empleos, reducción de pobreza e inclusión social mediante el desarrollo de nuevos productos, servicios y/o empresas con toque innovador que vengan a dar un valor agregado mediante la generación de competencia la cual permita el desarrollo de más unidades de negocios que sirvan como impulso en pro de mejorar los indicadores de un país.

\section{Importancia del emprendimiento en México}

Al hablar de la riqueza en materia económica en México se debe en gran medida a los aportes realizados por los emprendedores, no solo con sus contribuciones, si no con sus diferentes tipos de servicios y productos que en ocasiones son necesarios incluso para países en el extranjero y que han incentivado a generar mayores niveles de consumo y una mayor competencia que a su vez invita a ser superados, eso hace que las personas cada vez más busquen emprender y superarse a sî mismos y a la competencia ya existente (González, 2012).

Es importante recalcar que no solo es se trata de emprender, si no ofrecer experiencias, productos y servicios atractivos para el consumidor, de acuerdo con Schwab (2010) México es un país cuya economía es basada en la eficiencia, ya que el tamaño de mercado hace que sea atractivo para la generación de actividad emprendedora y esto a su vez hace que sus niveles de consumo sean altos y permitan a los dueños de nuevos negocios tener una mayor demanda de sus productos. 


\section{Factores de impacto}

Academia y generación de conocimiento para impulsar el emprendedurismo

Una de las variables a analizar es el impacto que tiene la academia a la hora de generar experiencia para poder comenzar con un proyecto, que si bien, los apoyos y herramientas existen en diferentes formas y medios, en ocasiones la carencia de enseñanza lo hace no tan localizables o fáciles de adquirir como se pudiera esperar (Wright y Hitt, 2017).

Staniewski (2016) menciona que la experiencia profesional y académica es el punto vivencial de las personas al obtener un grado de especialización determinado para realizar acciones de impacto que ayuden a la consecución de objetivos.

Ahora, la relación que guarda el factor experiencia con la variable emprendimiento es estrecha, existen estudios importantes que la consideran como determinante para poder tener éxito en este tipo de actividades. Un estudio realizado por Clarysse, Tartari y Salter (2011) menciona que existe una relación significativamente positiva entre el emprendimiento y la experiencia profesional y académica, esto en base una investigación que muestra que el $65 \%$ de las personas que emprenden un negocio tienen más probabilidad de éxito si cuentan con experiencia previa ya sea por medio de la academia o su pasado laboral, además durante la misma investigación se explica que el $30 \%$ de las personas que fallaron al intentar emprender lo atribuyen al desconocimiento de métodos y herramientas o simplemente por no tener el conocimiento de la industria en la cual quisieron aventurarse.

Otra investigación realizada por Stanewski (2016), realza aún más la importancia del factor de la experiencia, al mencionar que un $85 \%$ de las personas que cuentan con conocimiento previo laboral o académico en dicho país tienen un alto grado de probabilidad de éxito al momento de querer emprender y durante el mismo estudio, un 95\% de los encuestados consideran necesaria la experiencia para poder sobrevivir cuando se comienza un proyecto de emprendimiento.
Apoyos gubernamentales

El establecimiento de medidas de apoyo para las pequeñas y medianas empresas tiene como principal objetivo el impulsar el aprovechamiento de oportunidades, la creación de valor y la generación de empleo que estas puedan detonar. Sin embargo, no muchas personas tienen acceso a estos recursos principalmente por desconocimiento de dichos programas, estos apoyos principalmente impulsados por el gobierno tienen como misión el desarrollar los rubros económicos y sociales del país (Arping, Loranth y Morrison, 2018).

De acuerdo a Martínez, Ruiz y Biedma (2015) los apoyos gubernamentales se pueden definir como la consecuencia directa de querer dar un impulso a la economía local de un país, esto se deriva de muchas cuestiones, principalmente del empoderar, motivar y apoyar con distintos tipos de recursos a las personas para que puedan cumplir una metas en el ámbito del emprendimiento, para esto, también mencionan que antes de solicitar cualquier tipo de apoyo se tiene que contar con una base de lo que se quiere hacer y hacia donde se quiere llegar.

Por otro lado, si bien Canales, Rosa y Aldana (2017) mencionan que los apoyos gubernamentales pueden ser definidos como el método de incentivar y dar un respaldo a las personas que desean realizar una actividad emprendedora, concuerdan en que la mayor parte de las ocasiones las personas no los buscan principalmente por desconocimiento a su existencia, esto hace que los recursos que se ofrecen no se aprovechen de buena manera por la mayor parte de los emprendedores mexicanos.

Un estudio realizado por Valls, Cruz y Torruella (2012), muestra un dato importante, solo el $59 \%$ de las personas que se aventura a un proyecto de emprendimiento desconocen la existencia de instituciones como incubadores o fondeadoras que pudieran apoyarlos en su proyecto, más sin embargo un $94 \%$ de los evaluados considera que de conocer un mayor número de centro de capacitación como incubadoras o tuvieran mayor información sobre financiamientos $\mathrm{u}$ apoyos 
gubernamentales sus proyectos tendrían éxito, por lo que la relación de los factores de entorno con la variable de emprendimiento es muy cercana y significativamente positiva.

\section{Contribución a la economía}

No es un secreto que la economía del país es fuerte gracias a las Pequeñas y medianas empresas (PYMES), el INEGI (2016) menciona que el 97\% del gremio empresarial en México son PYMES, por lo que estas vienen a tener un detonante fuerte en la economía, y sin duda, hay quienes ven la práctica del emprendimiento como una forma de contribuir un poco en pro de beneficiar al país.

Podría pensarse un acto de patriotismo o simplemente una actividad para proteger la economía interna de las empresas foráneas, lo que es cierto es que el emprendimiento si genera un aporte al país. Iakovleva, Kolvereid y Stephan (2011) definen contribución a la economía como la actividad de una compañía en pro de generar la riqueza y bienestar de un país.

Por otro lado, Kardos (2012) define la contribución a la economía como la intención de aporte en pro de mejorar de manera considerable la economía de una nación atreves de la generación de aportes en la creación de empleos, etc. Por lo tanto, para concluir tomando en cuenta las definiciones anteriores, podemos decir que la contribución al país es el aporte que se hace en por de mejorar la calidad de vida en relación calidad de vida, económico y social.

\section{Educación}

La educación en materia de emprendimiento ha otorgado a los jóvenes la oportunidad de desarrollar factores que les permiten detectar oportunidades relevantes, sobre todo porque no es tarea sencilla encontrar algo, una falla, una pieza que pudiera faltar en un mundo que cada vez más pareciera que tiene todo bajo control, Un estudio elaborado por Bruton, et al. (2013) muestra la relación que existe entre la generación de emprendimiento y la enseñanza en esta rama aumenta la detección de oportunidades de nuevos negocios mostrando que hay una afectación de manera positiva al emprendimiento con un valor de $.787\left(\mathrm{R}^{2}\right)$ lo cual impacta en un $79 \%$ en la generación de actividades de emprendedurismo.

$\mathrm{Al}$ hacer referencia al nivel educativo y su relación con el emprendimiento es indudable pensar que entre más educación recibe una persona, esta tiene más posibilidades de emprender. Educación es definido por Valls, Cruz y Toruella (2012) como el grado de preparación de un individuo en busca de obtener una especialización en cierta materia de conocimiento y a su vez permitirle la detección de oportunidades.

Ahora bien, otro estudio muestra como la generación de emprendimiento se encuentra relacionada de manera significativamente positiva con la educación, ya que en los resultados de un método de investigación aplicado por Marulanda y Morales (2016) muestran un efecto medianamente aceptable a la hora de contar con un nivel educativo y emprender mostrando un valor de .372 R2 lo que implica que el nivel educativo impacta en un $37 \%$ en la generación de emprendimiento.

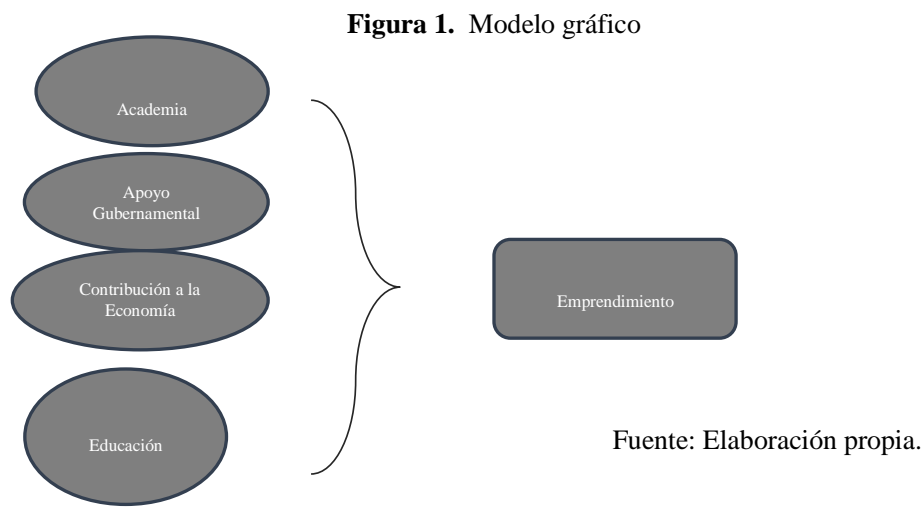


Finalmente, se puede entender en mayor medida lo que es el emprendimiento, sus características y que, a pesar de ser un concepto de amplias interpretaciones, se encuentran puntos clave que dan una idea clara de su importancia a nivel nacional e internacional.

La Figura 1 muestra una representación gráfica de las variables que se analizaron para la presente investigación y su importancia en la generación de emprendimiento.

\section{MÉTODO}

La metodología utilizada para el para el establecimiento del tipo de medición, se plantea el diseño desarrollado para la realización de la investigación, se delimita la muestra de la población a investigar, así como las características; finalmente se describe el instrumento, la recolección, análisis e interpretación de resultados.

\section{Enfoque y alcance de la investigación}

En relación con las características que establecen Hernández, Fernández y Baptista (2014) la investigación se desarrolla bajo un enfoque cuantitativo debido a que se recaban datos con la intención de analizar la información. De acuerdo con Abreu (2012), la investigación tendrá un alcance descriptivo; debido a las especificaciones de las propiedades y características de cada uno de los factores.
La investigación se realiza con diseño no experimental, de acuerdo con la inexistencia de modificación en las variables propuestas; por otro lado, será transeccional, debido a la implementación del instrumento de medición por única ocasión (Mendoza, 2010).

\section{Muestreo}

La unidad de análisis para esta investigación consiste en aquellos alumnos que han emprendido o buscan emprender un negocio, el análisis se realizara con dos campus de la escuela de negocios de la UANL con la finalidad de obtener resultados óptimos que soporten la encuesta a realizar. La intención es conocer los factores que conllevan a una persona a emprender y entender las diferencias principales entre ambos campus (Cuadro 1).

\section{Procedimiento de investigación}

El procedimiento de investigación que se utilizará para determinar los detonantes que generan emprendimiento en la escuela de negocios de la UANL y dos respectivos campos de esta como lo son el de Ciudad Universitaria y Sabinas Hidalgo N.L. Se aplicará mediante 32 preguntas, 13 de información general y el resto será de los factores analizados.

En el Cuadro 2 se muestra la distribución de preguntas utilizada y en la Cuadro 3 se plasma la escala empleada en las variables analizadas.

\section{Diseño de la investigación}

Cuadro 1. Selección de la muestra

\begin{tabular}{|c|c|}
\hline Característica & Número mínimo de estudiantes a encuestar \\
\hline $\begin{array}{c}\text { Inició negocio propio o tiene intenciones de emprender uno - } \\
\text { Encuestados Cd. Universitaria }\end{array}$ & 35 \\
\hline $\begin{array}{l}\text { Inició negocio propio o tiene intenciones de emprender uno - } \\
\text { Encuestados Sabinas Hidalgo } \\
\text { Fuente: Elaboración propia }\end{array}$ & 105 \\
\hline \multicolumn{2}{|c|}{ Cuadro 2. Distribución de preguntas por variable } \\
\hline Nombre de sección/factor & Secuencia de preguntas \\
\hline Información general & $1,2,3,4,5,6,7,8,9,10,11,12,13$ \\
\hline Academia & $14,15,16,17$ \\
\hline Apoyos gubernamentales & $18,19,20,21$ \\
\hline Contribución a la economía & $22,23,24,25$ \\
\hline Educación & $26,27,28,29$ \\
\hline Fuente: Elaboración propia. & \\
\hline
\end{tabular}


Cuadro 3. Procedimiento Likert.

\begin{tabular}{|c|c|c|c|c|}
\hline $\begin{array}{c}\text { Totalmente en } \\
\text { desacuerdo }\end{array}$ & En desacuerdo & $\begin{array}{c}\text { Ni de acuerdo ni en } \\
\text { desacuerdo }\end{array}$ & De acuerdo & $\begin{array}{c}\text { Totalmente } \\
\text { de acuerdo }\end{array}$ \\
\hline $\mathbf{1}$ & $\mathbf{2}$ & $\mathbf{3}$ & $\mathbf{4}$ & $\mathbf{5}$ \\
\hline
\end{tabular}

Fuente: Elaboración propia.

\section{ANÁLISIS DE RESULTADOS}

Para determinar los factores que detonan el emprendimiento en los estudiantes de la escuela de negocios de la UANL se diseñó un instrumento el cual se aplicó de manera directa en los Campus de Cd. Universitaria y Sabinas Hidalgo los cuales representaban el objeto de estudio. Durante este proceso de aplicación, se llenaron 140 encuestas las cuales permitieron completar el presente análisis y mostrar de manera directa los hallazgos realizados.

En este estudio, se recolecto información variada, siguiendo el patrón de que los individuos debían de pertenecer a la escuela de negocios de la UANL. En el campus de Sabinas Hidalgo fue donde se pudieron aplicar más cuestionarios $(74 \%)$ que en el campus de Cd. Universitaria (26\%), esta diferencia se centra en la disponibilidad por periodo de receso de ambos campus, no obstante, y afortunadamente, se obtuvo la participación necesaria para realizar este análisis a detalle y comparar la información.

Por otro lado, en el ámbito de carreras las más participativas y las cuales apoyaron de mayor manera con el presente estudio fueron la de contaduría pública (43\%) y administración (52\%) respectivamente, mientras que la carrera de tecnologías de información y negocios internacionales juntas participaron de manera muy mínima (5\%). Finalmente, con respecto al semestre actualmente cursando, los jóvenes de 9no semestre de las carreras mencionadas anteriormente fueron los que más colaboraron con el presente estudio (55\%).

En el Cuadro 4 se muestran a detalle las características de los individuos entrevistados, así como su respectiva distribución entre los campus y las carreras que representan.

Cuadro 4. Características generales de los individuos.

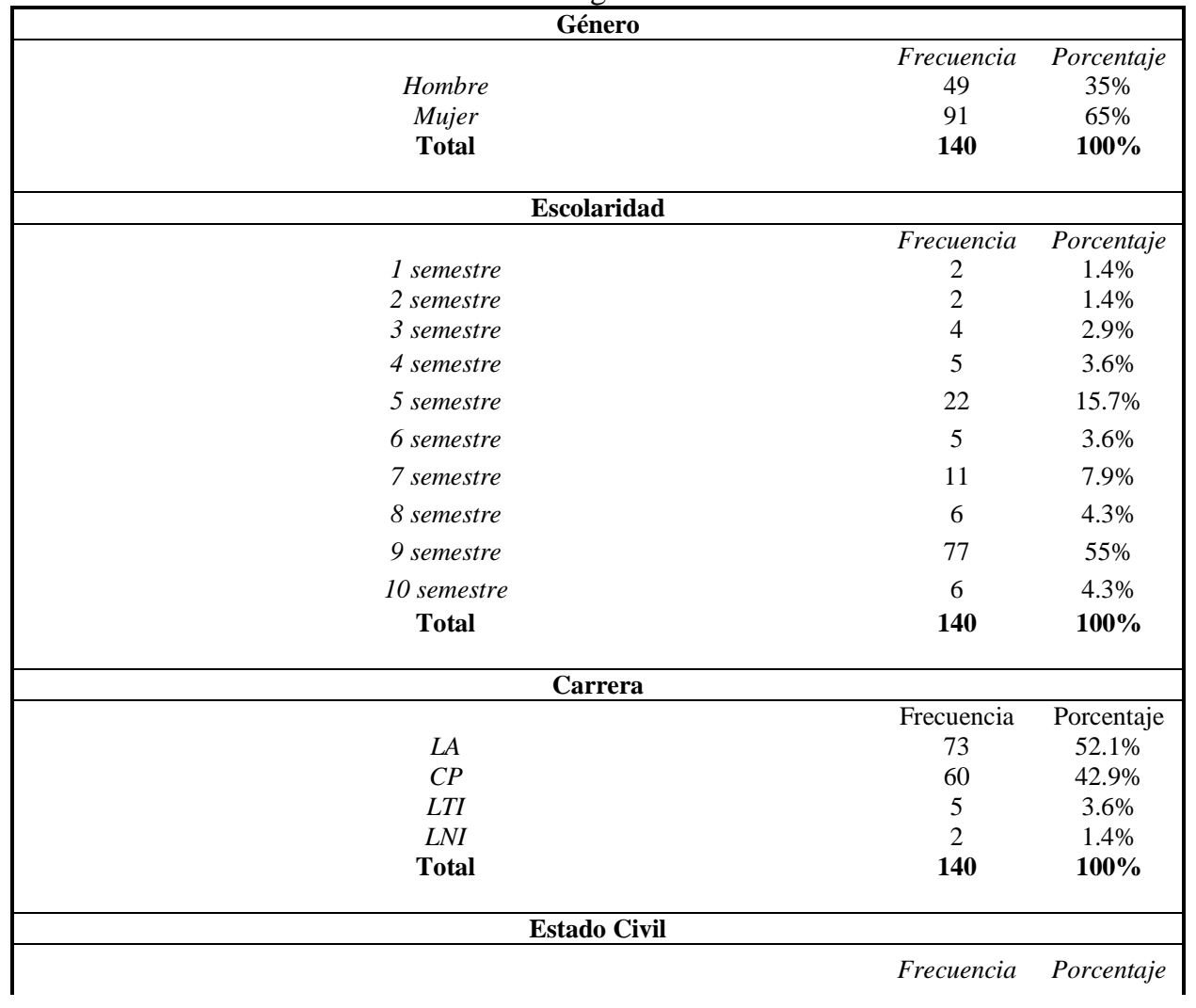




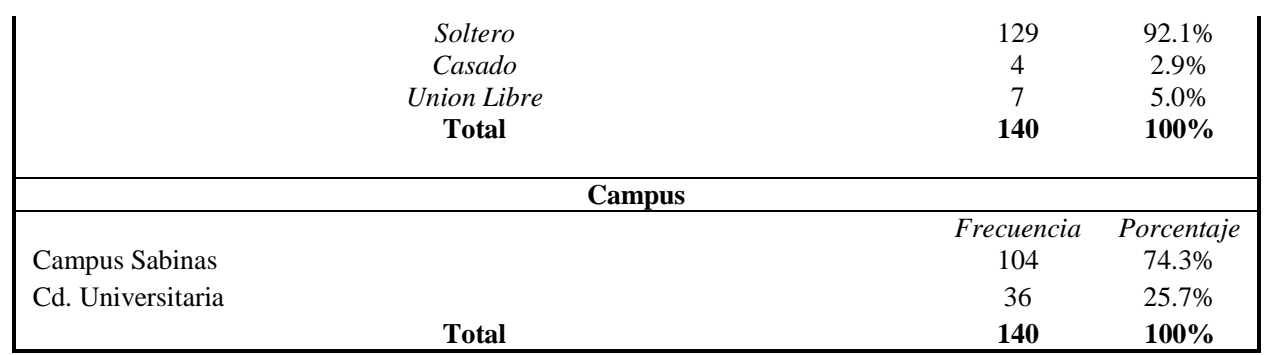

Nota: Resultados divididos en dos partes.

Fuente: Elaboración propia.

En la actualidad, la sociedad ha impulsado la creencia de una necesidad imperativa de que en algún punto de la vida de las personas se necesita emprender un negocio, ya sea para adquirir experiencia, probar nuevas oportunidades o simplemente por el hecho de contar con un ingreso adicional que permita a la persona subsistir en un país en el cual la vida constantemente se vuelve más compleja y retadora (Canales, Rosa y Aldana, 2017). Así mismo, esta practica a tenido a popularizarse en ciertos sectores de la población y cada día es más común ver el nacimiento de muchos pequeños negocios a lo largo del territorio nacional, desafortunadamente y de acuerdo con el INEGI, el 65\% de las empresas tiene un periodo de vida no mayor a los 8 años y tan solo en Nuevo León, la esperanza de vida de estos se reduce a un máximo de 7 años (INEGI, Censo Económico 2014).

En la Cuadro 5, se puede observar que si bien, una muy poca cantidad de los alumnos cuentan con un negocio propio (12\%), el deseo del resto que no cuentan con uno es comenzar con esta práctica $(98.4 \%)$.

Cuadro 5. Negocio actual y deseo de emprendimiento.

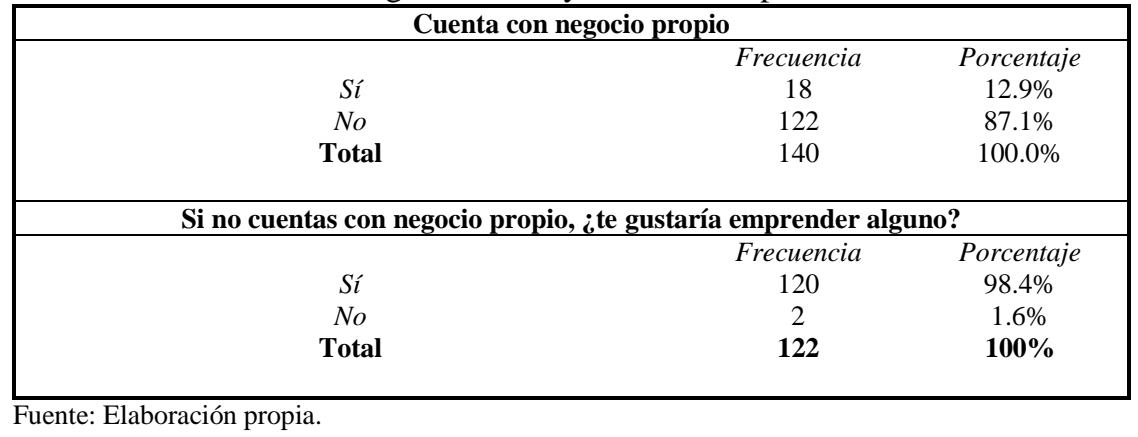

En las Gráfica 1 se muestra la preferencia de negocios sobre los cuales los estudiantes se decantarían a emprender, sorpresivamente la compra venta de productos se erigió como una de las más populares (25.7\%), esto seguido por el tema gastronómico, la venta de comida ha sido durante muchos años la practica más común de emprendimiento en la sociedad mexicana, en este estudio también se presentó como una de las predilectas de los alumnos
(20\%), también la parte de consultoría/despachos fue muy popular, ya que siendo en la mayoría de los jóvenes encuestados de la carrera de contador público el pensar en un despacho es una idea elemental de emprendimiento (19\%), por otro lado, también el con un establecimiento de consumo fue muy mencionado por los sujetos de esta investigación (18.8\%). 
Gráfica 1. Negocio deseado para emprender

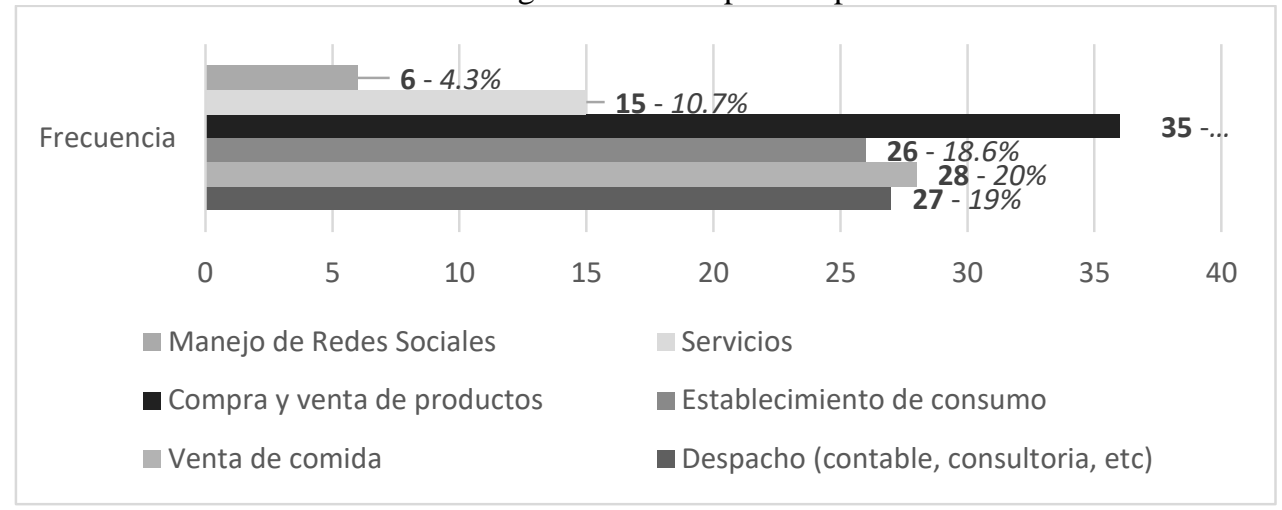

Fuente: Elaboración propia.

Mientras tanto, en la Gráfica 2 de factores de motivación para emprender, se muestra un dato único y contundente, ya que los estudiantes definen que para ellos la familia es uno de los impulsores principales para comenzar con esta práctica (62.1\%) mientras que por debajo se muestran otros factores como la carrera universitaria actualmente en curso (15\%).

Gráfica 2. Factores motivacionales

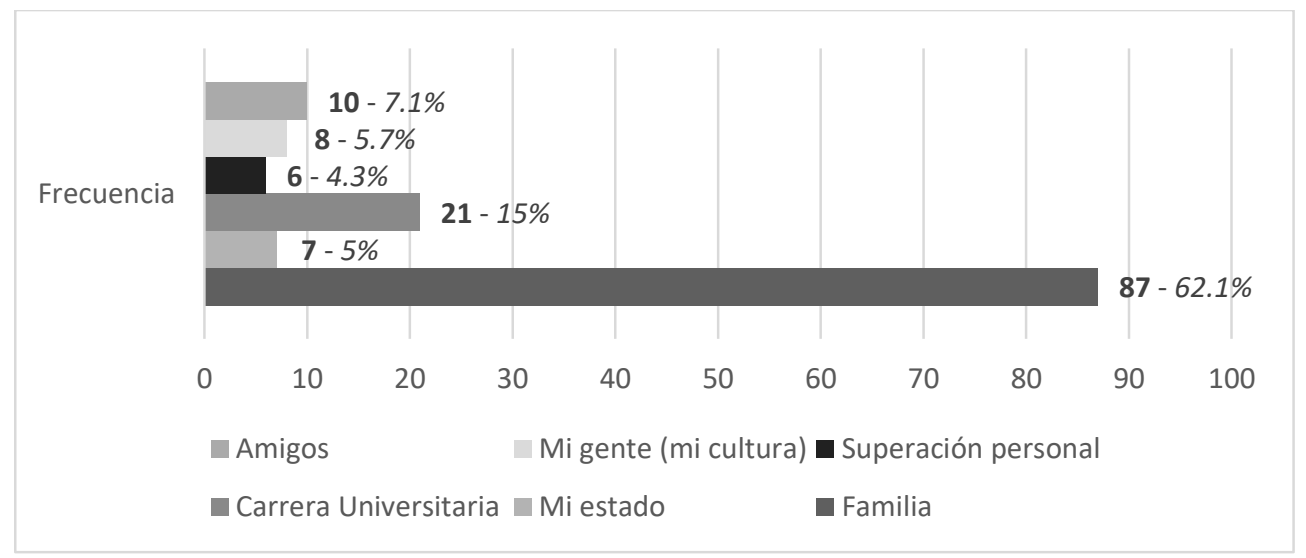

Fuente: Elaboración propia.

Sin duda, los hallazgos presentados en las gráficas anteriormente mostradas nos indican que los jóvenes efectivamente tienen la inquietud de emprender, que ven en la comercialización de productos, en el tema gastronómico y la consultoría una oportunidad de negocio que buscan capitalizar y que sobre todo la familia y su carrera universitaria son los motivantes principales que los impulsan a comenzar con esta práctica.

\section{Análisis}

El presente estudio mostro muchos hallazgos, puntos fuertes y áreas de oportunidad que se consideraran al momento de realizar las recomendaciones correspondientes, por lo pronto, es importante mencionar que el objetivo principal de la investigación se centra en encontrar aquellos detonantes en la población de las escuelas de negocios de la UANL que generen la actividad emprendedora, las 4 variables seleccionadas fueron propuestas con base a distintas investigaciones y seleccionados en base a un profundo análisis en el tema de emprendimiento.

En el Cuadro 6 se presenta un desglose completo de resultados que muestra las variables propuestas que detonan emprendimiento, así como el método utilizado. 
Cuadro 6. Factores que detonan la generación de emprendimiento

\begin{tabular}{|c|c|c|c|c|c|c|}
\hline Variable & Preguntas & $\begin{array}{l}\text { En } \\
\text { desacuerdo }\end{array}$ & $\begin{array}{l}\text { Totalmente } \\
\text { en } \\
\text { desacuerdo }\end{array}$ & $\begin{array}{l}\text { Ni de acuerdo } \\
\text { ni en } \\
\text { desacuerdo }\end{array}$ & $\begin{array}{l}\text { De } \\
\text { acuerdo }\end{array}$ & $\begin{array}{l}\text { Totalmente } \\
\text { de acuerdo }\end{array}$ \\
\hline & & 1 & 2 & 3 & 4 & 5 \\
\hline \multirow{4}{*}{$\underline{\text { Academia }}$} & $\begin{array}{l}\text { ¿Considera importante tener cursos, materias } \\
\text { o talleres en tu formación profesional para } \\
\text { emprender un negocio? }\end{array}$ & 0.7 & 0 & 3.6 & 26.4 & 69.3 \\
\hline & $\begin{array}{l}\text { ¿Considera que se debe tener una } \\
\text { preparación académica para la detección de } \\
\text { nuevas oportunidades de negocio? }\end{array}$ & 4.3 & 0.7 & 8.6 & 37.9 & 48.6 \\
\hline & $\begin{array}{l}\text { ¿Considera que el nivel de éxito al } \\
\text { emprender aumenta si en tu carrera } \\
\text { universitaria te enseñan como llevar a cabo el } \\
\text { emprendimiento? }\end{array}$ & 0.7 & 0.7 & 5.7 & 32.1 & 60.7 \\
\hline & $\begin{array}{l}\text { ¿Consideras que los profesores de tu } \\
\text { universidad deben enseñarte los medios para } \\
\text { emprender en lugar de prepararte para ser } \\
\text { empleado? }\end{array}$ & 0.7 & 0 & 10 & 30.7 & 58.6 \\
\hline \multirow{4}{*}{$\frac{\text { Contribución a la }}{\underline{\text { economía }}}$} & $\begin{array}{l}\text { ¿Considera usted que los emprendedores } \\
\text { aportan crecimiento a la economía de Nuevo } \\
\text { León? }\end{array}$ & 4.3 & 0 & 2.1 & 22.1 & 71.4 \\
\hline & $\begin{array}{l}\text { ¿Está de acuerdo en que la contribución al } \\
\text { Estado puede ser un detonante motivacional } \\
\text { para emprender un negocio? }\end{array}$ & 2.1 & 1.4 & 14.3 & 36.4 & 45.7 \\
\hline & $\begin{array}{l}\text { ¿Está de acuerdo en que la actividad de } \\
\text { emprendimiento genera un importante } \\
\text { porcentaje de la cantidad de empleos en el } \\
\text { Estado? }\end{array}$ & 1.4 & 0 & 7.9 & 26.4 & 64.3 \\
\hline & $\begin{array}{l}\text { ¿Le generaría motivación el saber que su } \\
\text { negocio podría contribuir a la generación de } \\
\text { empleo y el fortalecimiento de la economía? }\end{array}$ & 2.9 & 0 & 2.9 & 20 & 74.3 \\
\hline \multirow{4}{*}{$\begin{array}{l}\text { Apoyo } \\
\text { Gubernamental }\end{array}$} & $\begin{array}{l}\text { ¿Cuenta usted con conocimiento sobre } \\
\text { apoyos gubernamentales hacia los } \\
\text { emprendedores que apoyen en la generación } \\
\text { de nuevos negocios? }\end{array}$ & 20.7 & 2.9 & 24.3 & 32.1 & 20 \\
\hline & $\begin{array}{l}\text { ¿Considera usted que los apoyos brindados } \\
\text { en materia económica o financiamiento por } \\
\text { el gobierno son los adecuados para fomentar } \\
\text { la práctica del emprendedurismo? }\end{array}$ & 9.3 & 5.7 & 25 & 38.6 & 21.4 \\
\hline & $\begin{array}{l}\text { ¿Consideras que el gobierno brinda el apoyo } \\
\text { adecuado para dar apoyo a los } \\
\text { emprendedores o la gente que desee colocar } \\
\text { un negocio? }\end{array}$ & 20.7 & 7.9 & 35 & 20.7 & 15.7 \\
\hline & $\begin{array}{l}\text { ¿Considera que de contar con mayores } \\
\text { fuentes de financiamiento su nivel de éxito al } \\
\text { iniciar su proyecto hubiera mejorado? }\end{array}$ & 4.3 & 0 & 8.6 & 44.3 & 42.9 \\
\hline \multirow{4}{*}{ Educación } & $\begin{array}{l}\text { ¿Considera que se debe tener una } \\
\text { preparación académica para disminuir el } \\
\text { riesgo de fracaso en un negocio? }\end{array}$ & 2.1 & 1.4 & 30 & 35 & 31.4 \\
\hline & $\begin{array}{l}\text { ¿Considera que el nivel de estudios de una } \\
\text { persona influye en el éxito de un proyecto de } \\
\text { emprendimiento? }\end{array}$ & 13.6 & 2.9 & 30 & 25.7 & 27.9 \\
\hline & $\begin{array}{l}\text { ¿Considera que las universidades recalcan la } \\
\text { práctica del emprendimiento como } \\
\text { fundamental para el desarrollo económico de } \\
\text { un país? }\end{array}$ & 2.9 & 0 & 17.1 & 45 & 35 \\
\hline & $\begin{array}{l}\text { ¿Considera que las personas que egresan de } \\
\text { las universidades salen con la preparación } \\
\text { adecuada para emprender un negocio? }\end{array}$ & 7.9 & 2.9 & 25 & 40 & 24.3 \\
\hline
\end{tabular}

Fuente: Elaboración propia.

\section{Factor academia}

La academia es un factor que detona la práctica de emprendimiento, la relevancia que tiene para los estudiantes contar con materias, talleres y cursos sobre esta práctica (69.9\%) es fundamental, asimismo consideran que el nivel de éxito aumenta si se instruye con los pasos correctos para iniciar un negocio $(60.7 \%)$.

Por otro lado, los estudiantes también muestran la demanda de que los profesores deben anteponer el emprender sobre como inculcar al alumno el trabajar en una compañía $(58.7 \%)$ y derivado de esto, también se 
menciona que, si se cuenta con una preparación adecuada, se puede detectar con mayor facilidad las oportunidades de negocio $(48.6 \%)$

Es así como la academia se erige y se confirma su importancia por parte del alumnado como un medio de preparación esencial al momento de comenzar con la práctica del emprendimiento.

\section{Factor contribución a la economía}

El $74.3 \%$ de los encuestados se mosto motivado al saber que su negocio podría contribuir con el fortalecimiento de la economía y sobre todo con la generación de empleo (64.3\%), así mismo, también una gran mayoría considero como un aliciente el que su negocio pudiera apoyar de gran manera al estado de Nuevo León en materia económica (71.4\%).

Sin duda, el brindar nuestro apoyo en materia de crecimiento siempre fungirá como aliciente y más sobre todo en esta práctica que más allá de obtener beneficios propios, se busca contribuir con el desarrollo de miles de personas en el estado y sobre todo en el país.

\section{Apoyos gubernamentales}

Derivado de la presente investigación, este factor mostro opiniones dividas, por un lado, una parte de los encuestados (20.7\%) considera que el gobierno no brinda los apoyos necesarios para que los emprendedores realicen sus proyectos y otra parte en la misma proporción (20.7\%) menciona que, si los otorga, el resto simplemente se limitó a no estar de acuerdo ni en desacuerdo (35\%).

Otro hallazgo notorio e importante se derivó de que la gran mayoría de los estudiantes encuestados (87.2\%) afirma de manera positiva que de contar con mayores tipos de financiamientos por parte del gobierno su proyecto de emprendimiento aumentaría el nivel de éxito. Este dato se complementa cuando se cuestiona acerca de si se consideran adecuados los apoyos del gobierno, ya que en su mayoría (38.6\%) opinan que son suficientes, mas, sin embargo, y como se menciona, los estudiantes desearían contar con mayor número de créditos que les del soporte financiero requerido para comenzar con esta práctica.

\section{Educación}

Tal y como se vio con la academia, la tendencia con la educación es igual de positiva, ya que en su mayoría (35\%) consideran que es necesaria para disminuir riesgos o fracasos en esta práctica, mientras tanto, la presente investigación arroja que los estudiantes en su mayoría $(40 \%)$ considera que los jóvenes salen de la universidad con la preparación suficiente para emprender un negocio.

Existe también quienes se rehúsan a creer o a atribuir el éxito del emprendimiento a la educación, en uno de los cuestionamientos, una parte de los encuestados (13.9\%) considera que no se requiere y otro tanto en su mayoría (30\%) le es indiferente mencionado que no están ni de acuerdo ni en desacuerdo.

Sin duda alguna, podemos ver una inclinación al tema académico, contribución al estado y academia de manera positiva como detonantes de la práctica de emprendimiento, así mismo, los apoyos del gobierno aparecen como una solución a esta práctica, la cual se puede decir que contribuye de manera significativa a la realización de esta.

\section{DISCUSIÓN DE RESULTADOS}

El analizar los factores que generan emprendimiento en los estudiantes de las escuelas de negocios nos muestra como existe una percepción muy favorable acerca de cómo la academia, la educación y la contribución a la economía detonan esta práctica. Mas sin embargo la parte de apoyos gubernamentales muestra que existe un pequeño nivel de desconocimiento en este rubro derivado de las respuestas obtenidas, que, si bien al igual que los otros factores fue favorable, existen muchos puntos que nos da a la interpretación que se necesita reforzar el conocimiento en este tema.

Como recomendaciones derivadas de la presente investigación, se exhorta a los jóvenes a cuestionar de una manera objetiva y directa a la academia sobre el tema de financiamientos por parte del gobierno e 
inclusive de instituciones privadas hacia los emprendedores, se considera que esto traerá un complemento positivo a los resultados ya obtenidos sobre la línea de academia y educación y podrá aún más denotar el compromiso del gobierno de cada uno de los estados para con los emprendedores y sobre todo en mostrar el interés y las ganas de impulsar la economía del país.

\section{CONCLUSIONES}

El presente trabajo de investigación vino a corroborar puntos de gran importancia y sobre todo a generar nuevas interrogantes que nos hacen cuestionarnos si la educación que se imparte en materia de emprendimiento va por el camino correcto, porque de antemano sabemos que más allá de saber que el comerciar bienes y servicios ha sido una práctica que tiene siglos, ¿por qué los alumnos se decantan por esta actividad? ¿tendrán el conocimiento de los nuevos segmentos y oportunidades de mercado que brinda la era digital a la cual se ha incursionado en los últimos años? ¿se contará con la suficiente capacitación docente para permear en el alumno la detección de estas oportunidades? estos cuestionamientos vienen a detonar otro rubro que bien puede servir como una investigación a futuro y que generaría valor no solo a las escuelas de negocios de la UANL si no a las de todo el país.
Mientras tanto, también se tiene que tener en cuenta que el alumno en cuestión será un ente detonante en la generación de actividad económica en el país, por lo que inculcar en ellos la curiosidad y deseo de emprender debe ser una necesidad imperante en la práctica docente del país, si bien el experimentar nuevas oportunidades para generar experiencia en ellos es importante, también lo es el hacer referencia a que los tiempos han cambiado, que el incursionar en un mundo que es complejo y difícil como el de iniciar con un negocio propio es una experiencia necesaria en la formación profesional del estudiante.

Por último, el propósito de esta investigación más allá de encontrar las variables que generan emprendimiento busca ser una referencia no solo para las escuelas de negocios de la UANL, si no a mostrar que se necesita conocer la percepción de los alumnos de las universidades sobre esta práctica, ya que como se observa durante este trabajo y tomando como referencia datos del del senado de la republica del presente año, los emprendedores representan el $52 \%$ de la actividad económica del país, por lo que de manera necesaria es importante inculcar en los jóvenes el ánimo y deseo por comenzar que si bien es compleja, complicada y atarante, también es un muy gratificante no solo a razón personal del individuo, sino también en materia de desarrollo económico.

\section{Referencias}

Abreu, J. (2012). La pregunta de investigación: alma del método científico. Monterrey, México: UANL. Spenta México.

Alvarado, E., Antonio, C. y J. Ortiz. (2020). Agronegocios: ¿Qué piensan los jóvenes egresados de escuelas y facultades de negocios en México sobre el emprendimiento en el sector agropecuario? Revista Agro Productividad, 13(3), 37-46.

Álvarez, A., Giraldo, O., Martínez B. (2014). Emprendimiento, Factor Clave para el Crecimiento Económico. Escenarios: Empresa y Territorio, 3(1), 1-16.

Arping, S., Loranth, G., Morrison, A. (2009). Public Initiatives to support entrepreneurs: Credit Guarantees versus co-funding. Tinbergen Institute Discussion Paper. (9), 1-31. 
Bruton, G., Filatotchev, I., Si, S., Wright, M. (2013). Entrepreneurship and Strategy in Emerging Economies. Strategic Entrepreneurship Journal, vol 6, 169-180.

Canales, R., Ovando, W., Sanchez, R. (2017). Emprendimiento de la población joven en México, Una perspectiva critica. Entreciencias: diálogos en la sociedad del conocimiento, 12(1), ISSN: 2007-8064.

Canedo, J., Stone, D., Black, S., Lukaszewski, K. (2014). Individual Factors Affecting Entrepreneurship in Hispanics. Emerald Group Publishing Journal Articles, 101(1), 756-772.

Clarysse, B., Tartari, V., Salter, A. (2011). The impact of entrepreneurial capacity, experience and organizational support on academic entrepreneurship. ELSEVIER, vol 14, 1084-1093.

Connected Small Businesses US, The digital dividend for US small businesses (2017). Deloitte Consulting Research: The digital dividend for US small businesses. EUA. Recuperado de: https://www2.deloitte.com/content/dam/Deloitte/us/Documents/technology-mediatelecommunications/us-tmt-connected-small-businesses-Dec2017.pdf

Estadísticas Detalladas sobre las micro, pequeñas y medianas empresas. (2016). Boletín Informativo Numero 285/16: Discover human anatomy. Aguascalientes, México. Instituto Nacional de Estadística Geográfica e Informática (INEGI). Recuperado de: http://www3.inegi.org.mx/rnm/index.php/catalog/330/download/9792

Hernandez. R., Fernández y C., Baptista. (2014). Metodología de la Investigación. México: McGraw Hill.

Hochsztain, E. y Messina, M. (2015). Factores de Éxito de un emprendimiento: Un estudio exploratorio con base en técnicas de data mining. Revista TEC Empresarial, 9(1), 31-40.

Kardos, M. (2012). The Relationship between Entrepreneurship, Innovation and sustainable Development. ELSEVIER, 1030-1035.

Marulanda, F., Morales, S. (2016). Entorno y Motivaciones para emprender. Revista. Escuela de Administración de Negocios, 81(1), 12-28.

Martínez, S., Biedma, J. y Ruiz, J. (2015). Las condiciones del entorno emprendedor y el desarrollo económico: un análisis de los países GEM. Revista de Economía Mundial. (41), 181-21.

Minniti, M. (2012). El Emprendimiento y el Crecimiento Económico de las Naciones. Department of Strategy and Enterprenuership, COX School of Business Journal.

Schwab, K. (2010). The Global Competitiveness Report 2010-2011. Geneve, Swiss. Retrieved October 15, 2010 from World Economic Forum Web Site http://www3.weforum.org/docs/WEF GlobalCompetitivenessReport 2010-11.pdf

Staniewski, M. (2016). The Contribution of Business Experience and Knowledge to Successful Entrepreneurship. Journal of the University of Finance and Management in Warsaw, 1-32.

Valls, J.,Cruz, C., Torruella, A., Juanes, E., Canessa, M. y Hormiga, E. (2012). Causas de Fracaso de los emprendedores. Netbiblio, España.

Wright, M., Hitt, M. (2017). Strategic Entrepreneurship and SEJ: Development and Current Progress Strategic Entrepreneurship Journal, 11(1), 200-210. 